\title{
An Odyssey into the Land of Deleterious Rare Mutations in Colorectal Liver Metastases
}

\author{
Timothy E. Newhook, MD, Yun Shin Chun, MD, and Jean-Nicolas Vauthey, MD \\ Department of Surgical Oncology, The University of Texas MD Anderson Cancer Center, Houston, TX
}

In this issue, Kobayashi et al. present the survival of patients with $B R A F$ V600E mutations who underwent initial hepatectomy for colorectal liver metastases (CLM) from 2005 to 2016 at six high-volume institutions in Japan. ${ }^{1}$ Among a total of 33 patients in this multicenter, retrospective, cohort study, only $21.2 \%$ received preoperative chemotherapy, and $51.5 \%$ received adjuvant chemotherapy. The authors reported 1- and 3-year recurrence-free survival (RFS) rates of $24.2 \%$ and $6.1 \%$, respectively, after median post-hepatectomy follow-up of 49.2 months. The majority of patients (93.9\%) developed recurrence at a median of 5.3 months, with $42.4 \%$ recurring in multiple sites and $87.9 \%$ in an unresectable pattern. Median overall survival (OS) for the study cohort was 31.1 months, including six patients who survived 5 years. The Japanese approach to patients with resectable CLM does not typically include the use of preoperative chemotherapy outside of a clinical trial, as noted by the authors. Thus, this small cohort reflects the natural history of BRAF V600E mutations for patients who undergo CLM resection without patient selection based on response to preoperative chemotherapy as a surrogate marker for tumor biology.

Among all patients with metastatic colorectal cancer, approximately $10 \%$ have BRAF mutations, which are associated with aggressive disease and worse oncologic outcomes. ${ }^{2} B R A F$ mutations are rare in patients undergoing CLM resection, occurring in 2-6\% of hepatectomy series.

(C) Society of Surgical Oncology 2020

First Received: 11 June 2020

Accepted: 28 June 2020;

Published Online: 10 July 2020

J.-N. Vauthey, MD

e-mail: jvauthey@mdanderson.org
An initial report from Memorial Sloan Kettering Cancer Center demonstrated that metastatic colorectal cancer patients with $B R A F$ mutations $(n=91)$ are less likely to harbor liver-limited disease compared with $B R A F$ wildtype patients $(n=423)$, and consequently, undergo CLM resection less frequently. ${ }^{2}$ More recently, Gagnière and colleagues reported a multi-institutional experience of 1497 patients who underwent hepatectomy for CLM, with $B R A F$ mutations occurring in $2 \%$ of patients. Median RFS after hepatectomy was 10 months for $B R A F$ mutant patients, compared with 20 months for $B R A F$ wild-type. ${ }^{3}$

The most frequent $B R A F$ mutation is a substitution in codon 600 , resulting in an amino acid change from valine (V) to glutamic acid (E). Studies have shown conflicting results on the prognostic impact of non-V600E mutations, due to small sample sizes. In the study by Gagnière et al., among 35 patients with BRAF mutations, 25 (71\%) had V600E mutations, which was not associated with worse survival than non-V600E mutations. ${ }^{3}$ In contrast, Margonis and colleagues reported worse survival after CLM resection with $B R A F$ V600E mutations but not with non-V600E mutations. However, this study included only 33 patients with V600E and 6 patients with non-V600E mutations, and therefore, the numbers are too small to draw reliable conclusions. $^{4}$

In our preliminary experience of 20 patients with $B R A F$ mutations who underwent hepatectomy from 2006 to 2015, we found no significant differences in either RFS or OS between V600E and non-V600E mutations. ${ }^{5}$ Among these 20 patients, $90 \%$ received preoperative chemotherapy. Compared with a control group of $B R A F$-mutated patients treated with chemotherapy alone for isolated hepatic metastases, median OS rates were significantly better with surgery (46 months) than with chemotherapy alone (19 months). Our median OS of 46 months contrasts with that reported by Kobayashi et al. of 31.1 months, 
underscoring the importance of preoperative therapy to select patients for surgery. Recent studies suggest improved survival with systemic therapy, including firstline FOLFOXIRI plus bevacizumab. ${ }^{6}$ In the second-line setting, the BEACON trial, noted by Kobayashi et al., demonstrated improved response rates and survival with the triplet regimen of encorafenib, cetuximab, and binimetinib. ${ }^{7}$ For patients considered for CLM resection, the duration of preoperative systemic therapy should not exceed 2-3 months to avoid hepatotoxicity, particularly with FOLFOXIRI.

The genetic landscape of colorectal cancer is dominated by three somatic mutations: TP53, APC, and RAS (Fig. 1). While attention has recently been given to these common mutations in doublet or triplet combinations, it is important to recognize the independent, deleterious effects of single, rare mutations on CLM prognosis. ${ }^{8,9}$ As tasked by Dr. Moertel in his Karnofsky memorial lecture, understanding rare pathologies is our responsibility as specialists in oncology. ${ }^{10}$ In addition to $B R A F$ in the mitogen-activated protein (MAP) kinase pathway, other infrequent but critical gene mutations on other pathways determine survival in patients with resectable and unresectable metastatic colorectal cancer. These include SMAD4 in the transforming growth factor- $\beta$ (TGF- $\beta$ ) pathway and $F B X W 7$ in the Notch pathway. We identified SMAD4 mutations in $13 \%$ of patients with both resectable and unresectable CLM, which suggests that $S M A D 4$, unlike $B R A F$, does not affect the rate of liver-limited disease. ${ }^{11}$ For patients with resectable and unresectable CLM, 3-year and 5-year OS rates were $62 \%$ and $22 \%$, respectively, with SMAD4 mutations compared with $82 \%$ and $38 \%$ with SMAD4 wild-type. More recently, our group examined $F B X W 7$ mutations, occurring in $6 \%$ of resected CLM, which were associated with significantly worse 5-year OS than FBXW7 wild-type (30\% vs. $61 \%){ }^{12}$ Performing mutational panels to include rare mutations, such as these, provides a window into individual patient tumor biology and may have tremendous implications for

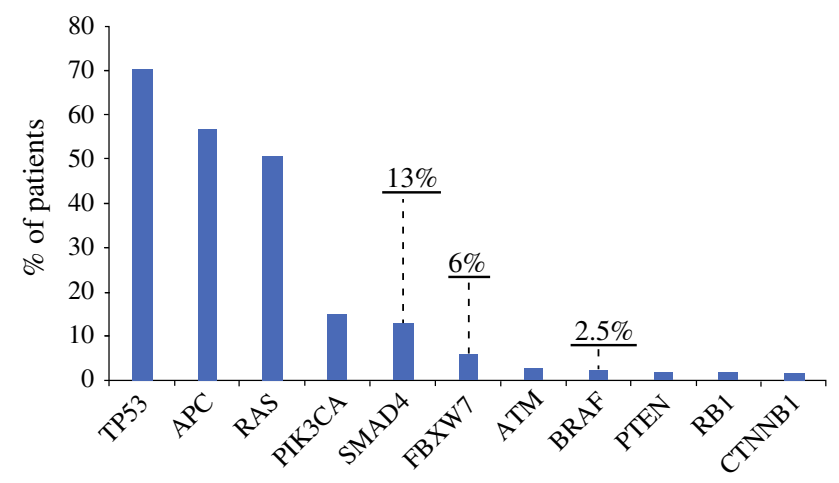

FIG. 1 Genetic landscape of colorectal cancer, including rare deleterious mutations future decisions on treatment sequencing, re-resection after recurrence, resection when faced with extensive or extrahepatic disease, as well as liver transplantation for patients with CLM.

We congratulate Kobayashi and colleagues for their multicenter report, which provides a clear view of the deleterious impact of BRAF V600E mutations on oncologic outcomes for patients with resected CLM. Surgeons must certainly be aware of the presence or absence of $B R A F$ V600E mutations when selecting patients for hepatectomy or planning treatment sequencing. Rapidly emerging data highlight the importance of the broader landscape of gene mutations in determining survival after CLM resection. One of the looming questions is how large a gene panel should be considered. Before making surgical decisions for CLM patients, we recommend testing for mutations in RAS, BRAF, TP53, SMAD4, FBXW7, and HER 2 and determination of microsatellite instability. The mutational status of these genes has implications for decision-making during and following the complex and sequential treatments of patients with CLM.

DISCLOSURES The authors declare no conflicts of interest.

\section{REFERENCES}

1. Kobayashi S, Takahashi S, Takahashi N, et al. Survival outcomes of resected BRAF V600E mutant colorectal liver metastases: a multicenter retrospective cohort study in Japan. Ann Surg Oncol. 2020. https://doi.org/10.1245/s10434-020-08817-8.

2. Yaeger R, Cercek A, Chou JF, et al. BRAF mutation predicts for poor outcomes after metastasectomy in patients with metastatic colorectal cancer. Cancer. 2014;120(15):2316-24.

3. Gagniere J, Dupre A, Gholami SS, et al. Is hepatectomy justified for BRAF mutant colorectal liver metastases? A multi-institutional analysis of 1497 patients. Ann Surg. 2020;271(1):147-54.

4. Margonis GA, Buettner S, Andreatos N, et al. Association of BRAF mutations with survival and recurrence in surgically treated patients with metastatic colorectal liver cancer. JAMA Surg. 2018;153(7):e180996.

5. Cloyd J, Tzeng C, Mizuno T, Conrad C, Omichi K, Aloia T, Vauthey J, Chun Y. Braf mutation is not a contraindication to resection of colorectal liver metastases. $H P B$. 2017;19(S1): S55-6.

6. Cremolini C, Loupakis F, Antoniotti C, et al. FOLFOXIRI plus bevacizumab versus FOLFIRI plus bevacizumab as first-line treatment of patients with metastatic colorectal cancer: updated overall survival and molecular subgroup analyses of the openlabel, phase 3 TRIBE study. Lancet Oncol. 2015;16(13):1306-15.

7. Kopetz S, Grothey A, Yaeger R, et al. Encorafenib, binimetinib, and cetuximab in BRAF V600E-mutated colorectal cancer. $N$ Engl J Med. 2019;381(17):1632-43.

8. Chun YS, Passot G, Yamashita S, et al. Deleterious effect of RAS and evolutionary high-risk TP53 double mutation in colorectal liver metastases. Ann Surg. 2019;269(5):917-23.

9. Kawaguchi Y, Kopetz S, Newhook TE, et al. Mutation status of RAS, TP53, and SMAD4 is superior to mutation status of RAS alone for predicting prognosis after resection of colorectal liver metastases. Clin Cancer Res. 2019;25(19):5843-51. 
10. Moertel CG. Karnofsky memorial lecture. An odyssey in the land of small tumors. J Clin Oncol. 1987;5(10):1502-22.

11. Mizuno T, Cloyd JM, Vicente D, et al. SMAD4 gene mutation predicts poor prognosis in patients undergoing resection for colorectal liver metastases. Eur J Surg Oncol. 2018;44(5):684-92.

12. Kawaguchi Y, Newhook TE, Vreeland TJ, et al. 438 Alteration of $F B X W 7$ is associated with worse survival in patients undergoing resection of colorectal liver metastases. Gastroenterology. 2020;158(6):S-1511.

Publisher's Note Springer Nature remains neutral with regard to jurisdictional claims in published maps and institutional affiliations. 